\title{
Comparative Study of Absorbing Boundary Conditions for the Time-Domain Beam Propagation Method
}

\author{
Jun Shibayama, Tomokazu Takahashi, Junji Yamauchi, Member, IEEE, and Hisamatsu Nakano, Fellow, IEEE
}

\begin{abstract}
Various absorbing boundary conditions (ABCs) are compared in the analysis of the time-domain finite-difference beam propagation method. For a one-dimensional problem, the following ABCs are tested: Higdon's absorbing boundary, Ramahi's complementary operators method (COM), its concurrent version (C-COM) and Bérenger's perfectly matched layer (PML). It is found that the second- and third-order C-COMs with three and four boundary cells are comparable to the PMLs with eight and 16 cells, respectively. The effectiveness of the C-COM is also discussed in a two-dimensional problem.
\end{abstract}

Index Terms-Absorbing boundary conditions, beam propagation method, time-domain analysis.

\section{INTRODUCTION}

$\mathbf{T}$ HE STRUCTURE of an optical waveguide is, in general, unbounded. However, simulation of an optical waveguide must be performed within a finite computational window, due to limited computational resources. Therefore, it is necessary to impose an appropriate boundary condition that absorbs outgoing waves at the computational window edges. For the beam propagation method (BPM), a transparent boundary condition (TBC) has widely been employed [1]. In addition, Higdon's absorbing boundary condition (HABC) [2], corresponding to a uniform absorbing boundary condition [3], [4], has been applied to the BPM. The TBC and HABC can directly be incorporated into a tridiagonal matrix that appears in the BPM based on the conventional Crank-Nicholson $(\mathrm{CN})$ scheme, leading to no increase in the computational cost. A perfectly matched layer (PML) [5] was also tested in [3], [4], [6]. Although the PML shows an improved performance compared to earlier techniques, it usually requires many boundary cells, resulting in an increase in the computational cost.

Recently, Ramahi proposed a complementary operators method (COM) [7] and its concurrent version (C-COM) [8] for the finite-difference time-domain (FDTD) method. The $\mathrm{C}$-COM has the advantage that the number of boundary cells can be reduced to only a few, maintaining a dynamic range comparable to that of the PML [9]. Several authors incorporated the COM and C-COM into the BPM and discussed their advantages [10]-[12].

The conventional BPM is formulated in frequency domain. To treat a transient behavior, another time-domain scheme,

Manuscript received November 27, 2000.

The authors are with the College of Engineering, Hosei University, Koganei, Tokyo 184-8584, Japan.

Publisher Item Identifier S 1041-1135(01)03094-4. a so-called TD-BPM, has been developed based on a slowly varying envelope approximation in time [13]-[16]. For the TD-BPM, the TBC [14] and PML [16] have been used so far. However, the COM and C-COM have not been applied to the TD-BPM. Furthermore, no literature has discussed a detailed comparison among the above mentioned $\mathrm{ABCs}$ for the TD-BPM.

The purpose of this letter is to incorporate various $\mathrm{ABCs}$ into the TD-BPM based on the FD technique, and compare their performances. For a one-dimensional (1-D) problem, the following ABCs are tested: HABC, COM, C-COM and PML. To assess these conditions, an optical pulse propagating through a uniform media is investigated. It is found that the C-COMs with three and four boundary cells are comparable to the PMLs with eight and 16 cells, respectively. The effectiveness of the C-COM is also demonstrated for the two-dimensional (2-D) TD-BPM [15].

\section{DISCUSSION}

A time-domain Fresnel equation [13]-[16] is solved by the $\mathrm{CN}$ scheme, in which the tridiagonal matrix is generated.

For the HABC, a time dependence of the field, $\exp (-j \omega t)$, is assumed in the derivation of the boundary condition, so that the following boundary operator is obtained:

$$
B_{N} E=\prod_{i=1}^{N}\left[\partial_{x}+\frac{\xi_{i}}{c} \partial_{t}+\left(\alpha_{i}-\frac{j \omega \xi_{i}}{c}\right)\right] E=0
$$

where $N$ is the number of the order, $c$ is the speed of light in a vacuum, $\xi_{i}=\cos \theta_{i}$, and $\alpha_{i}$ is the parameter introduced for stability. Throughout this letter, we choose $\theta_{i}=0$ and $\alpha_{i}=0$. We denote the $N$ th-order $\operatorname{HABC}$ as $\operatorname{HABC} N$, and investigate $\mathrm{HABC} 1, \mathrm{HABC} 2$ and HABC 3 (the HABC1 corresponds to the TBC in frequency domain, when an adaptive procedure is introduced [4]). The numbers of cells required to implement the HABCs are two, three and four, respectively. Note that for the $\mathrm{HABC} 2$ and $\mathrm{HABC} 3$, the systems are no longer tridiagonal on the first and last rows in their matrices. Fortunately, the tridiagonal structure can easily be recovered by linear combinations [3]. Hence, there is no increase in the computational time, as long as the HABCs are used.

With respect to the COM and C-COM, the HABCs are used to make complementary operators whose reflection coefficients 


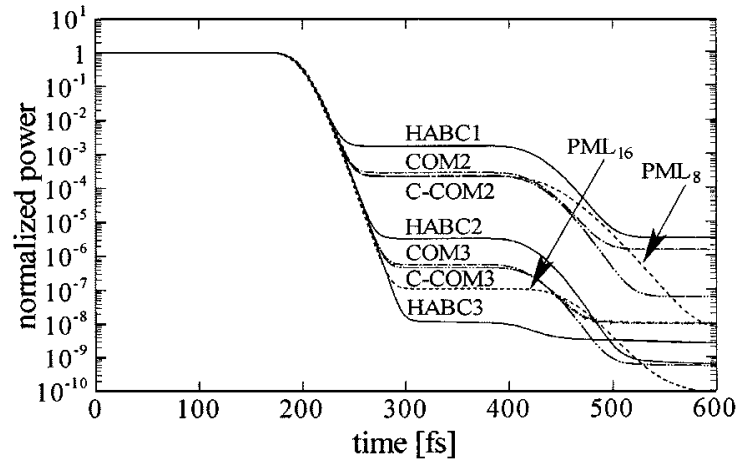

Fig. 1. Time evolution of the normalized remaining power.

have the same amplitude with opposite phase. The two complementary operators for the TD-BPM are expressed as

$$
\begin{aligned}
B_{N}^{-} E & =\partial_{x} \prod_{i=1}^{N-1}\left[\partial_{x}+\frac{\xi_{i}}{c} \partial_{t}+\left(\alpha_{i}-\frac{j \omega \xi_{i}}{c}\right)\right] E \\
& =0 \\
B_{N}^{+} E & =\left(\partial_{t}-j \omega\right) \prod_{i=1}^{N-1}\left[\partial_{x}+\frac{\xi_{i}}{c} \partial_{t}+\left(\alpha_{i}-\frac{j \omega \xi_{i}}{c}\right)\right] E \\
& =0 .
\end{aligned}
$$

The disadvantage of the COM is that the computational time is twice that of the $\mathrm{HABC}$, since the $\mathrm{COM}$ requires two independent solutions of the whole computational region using (2) and (3). On the other hand, the C-COM has the advantage that the two independent solutions are applied only to a narrow boundary region that surrounds an internal computational region. Therefore, the $\mathrm{C}-\mathrm{COM}$ is more efficient than the COM. Here, we use the second- and third-order conditions (COM2, COM3, C-COM2 and C-COM3). For the C-COM2 and $\mathrm{C}-\mathrm{COM} 3$, the numbers of cells required for the boundary region are taken to be three and four, respectively.

For the PML, we test two cases where the absorbing regions are composed of eight and 16 cells. We have empirically optimized a conductivity profile and a theoretical reflection coefficient $R(0)$. For eight cells, a square profile with $R(0)=10^{-2}$ is used, while for 16 cells, a cubic profile with $R(0)=10^{-4}$ is used.

To understand the fundamental characteristics of the $\mathrm{ABC}$, we treat a 1-D problem in which two computational edges are terminated with the ABC. The optical pulse of the Gaussian profile with a $1 / e$ width of $4 \mu \mathrm{m}$ propagates through the uniform media with a refractive index of $n=2$. We calculate the time evolution of the remaining power in the computational region. The spatial sampling width and time step $\Delta t$ are fixed to be 0.1 $\mu \mathrm{m}$ and $1 \mathrm{fs}$, respectively.

Fig. 1 shows the time evolution of the normalized remaining power (a wavelength is chosen to be $\lambda=1.55 \mu \mathrm{m}$ throughout this letter). The propagating field is absorbed by the $\mathrm{ABC}$ at the computational edge after $200 \mathrm{fs}$. The field, generated by the first-order reflection, is absorbed by the $\mathrm{ABC}$ at the opposite

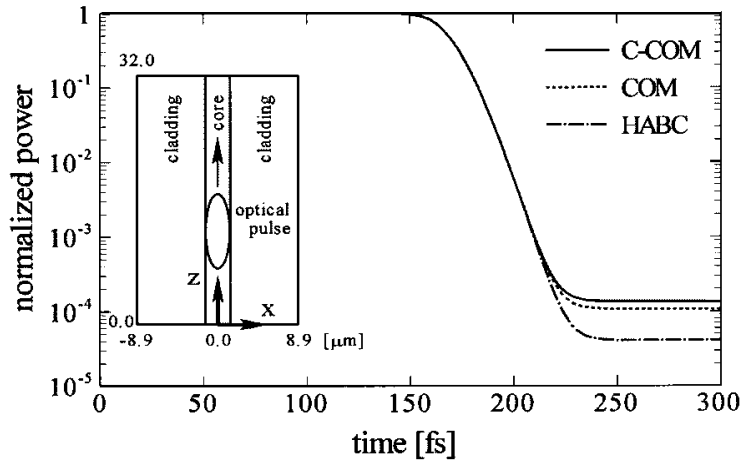

Fig. 2. Time evolution of the normalized remaining power for pulse propagation in a slab waveguide.

edge after $400 \mathrm{fs}$. The remaining power after $500 \mathrm{fs}$ is caused by the second-order reflection.

As expected, the accuracy of the HABC increases with an increase in the order of the HABC. In this analysis, the HABC2 and $\mathrm{HABC} 3$ show good performances with a remaining power of less than $10^{-8}$ at $500 \mathrm{fs}$. For suppressing the first-order reflection, the performance of the COM is almost identical to that of the $\mathrm{C}-\mathrm{COM}$. This is because the COM and $\mathrm{C}-\mathrm{COM}$ are based on the same HABC. However, owing to the difference in an averaging technique between the two methods [8], the C-COM is more effective for suppressing the second-order reflection than the COM. It is noteworthy that the C-COM2 with three boundary cells is comparable to the PML with eight cells. It is also seen that the C-COM3 with four cells compares with the PML with 16 cells.

As discussed above, the HABC and C-COM can reduce the computational memories required for an $\mathrm{ABC}$ to substantial degree, maintaining acceptable accuracy. This fact becomes more significant for a higher dimensional problem. Therefore, consideration is next given to the applications of the HABC and $\mathrm{C}-\mathrm{COM}$ to the 2-D TD-BPM based on the alternating-direction implicit method (ADIM) [15].

Note that for the ADIM algorithm, the HABC cannot be uniformly applied to the boundaries with respect to the transverse $(x)$ and longitudinal $(z)$ directions. In the first step of the ADIM, therefore, the HABC is applied to the boundary in the $x$ direction and is treated implicitly as in the case of a 1-D problem. For the boundary in the $z$ direction, the HABC can be treated explicitly. In the second step, the procedures treating the $x$ and $z$ directions are carried out interchangeably. These procedures are extended to the COM and C-COM. We here test the HABC, $\mathrm{COM}$ and $\mathrm{C}-\mathrm{COM}$ based on the second-order conditions. The number of boundary cells for the C-COM is set to be four.

First, we investigate the case for the normal incidence to the computational edge. We analyze the slab waveguide whose refractive indices of the core and cladding are 1.75 and 1.7, respectively. The core width is $2 d \simeq 1.78 \mu \mathrm{m}$. The sampling widths are $\Delta x=d / 6 \simeq 0.15 \mu \mathrm{m}$ and $\Delta z=0.1 \mu \mathrm{m}$. The time step is chosen to be $\Delta t=1 \mathrm{fs}$. The incident field is the pulse having the fundamental mode profile in $x$, and the Gaussian profile with a $1 / e$ width of $4 \mu \mathrm{m}$ in $z$. Fig. 2 shows the time evolution of the normalized power remaining in the computational region. It is seen that the accuracy of the HABC is slightly better than those 


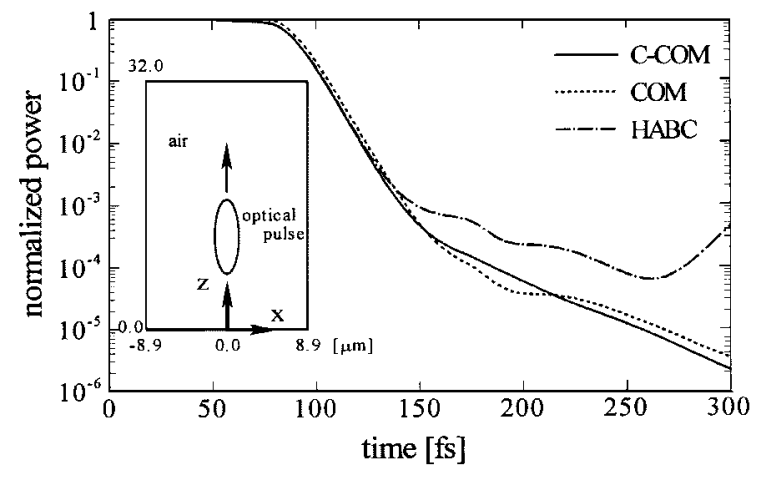

Fig. 3. Time evolution of the normalized remaining power for Gaussian pulse propagation in free space.

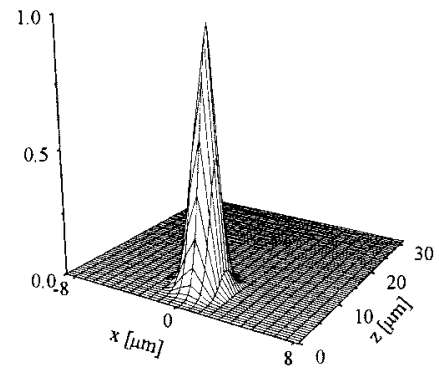

(a)

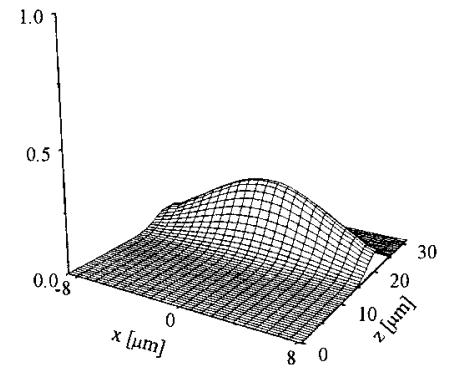

(b)

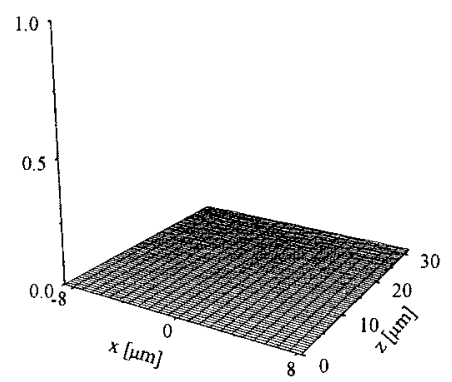

(c)

Fig. 4. Time evolution of the Gaussian pulse in free space: (a) $t=0 \mathrm{fs}$, (b) $t=50 \mathrm{fs}$ and (c) $t=150 \mathrm{fs}$. The fields are displayed only in the internal computational region.

of the COM and C-COM. This attributes to the fact that the conditions of the second-order COM and C-COM are analogous to that of the first-order HABC.

It should be noted that the COM and C-COM are effective for absorbing the outgoing waves with divergence angles, when compared to the HABC [10]-[12]. To clarify this fact in the TD-BPM, we next analyze a Gaussian pulse propagating in free space. The pulse to be analyzed consists of the Gaussian profile with $1 / e$ widths of 2 and $4 \mu \mathrm{m}$ in $x$ and $z$, respectively. The numerical parameters are the same as those used in Fig. 2. In Fig. 3, it is found that the C-COM works better than the HABC. An increase in the remaining power observed for the HABC after $250 \mathrm{fs}$ is due to instability, which can be eliminated by an appropriate choice of $\alpha$ in (1).

Fig. 4 illustrates the time evolution of the Gaussian pulse, in which (a), (b), and (c) are at $t=0,50$, and $150 \mathrm{fs}$, respectively. The boundaries are terminated with the C-COM. The fields impinging on the boundaries are effectively absorbed by the C-COM, and the reflected wave from the boundaries cannot be observed at $t=150 \mathrm{fs}$.

\section{CONCLUSION}

We have applied several ABCs to the TD-BPM. For a 1-D problem, the HABCs offer good performances without an increase in the computational cost. In spite of less boundary cells, the second- and third-order C-COMs are comparable to the PMLs with eight and 16 cells, respectively. We next extend the HABC and C-COM to the 2-D TD-BPM. It is shown that the C-COM effectively absorbs the outgoing waves with divergence angles, when compared to the HABC.

\section{ACKNOWLEDGMENT}

The authors would like to thank T. Ando and R. Shoji for their invaluable discussions.

\section{REFERENCES}

[1] G. R. Hadley, "Transparent boundary condition for the beam propagation," Opt. Lett., vol. 16, no. 9, pp. 624-626, 1991

[2] R. L. Higdon, "Absorbing boundary conditions for difference approximations to the multidimensional wave equation," Math. Comput., vol. 47, no. 176 , pp. 437-459, 1986.

[3] C. Vassallo and F. Collino, "Highly efficient absorbing boundary conditions for the beam propagation method," J. Lightwave Technol., vol. 14, pp. 1570-1577, June 1996.

[4] _ "Highly efficient transparent absorbing boundary conditions for finite difference beam propagation method at order four," J. Lightwave Technol., vol. 15, pp. 1958-1965, Oct. 1997.

[5] J. P. Bérenger, "A perfectly matched layer for the absorption of electromagnetic waves," J. Comput. Phys., vol. 114, pp. 185-200, 1994.

[6] W. P. Huang et al., "The perfectly matched layer (PML) boundary condition for the beam propagation method," IEEE Photon. Technol. Lett., vol. 8, pp. 649-651, May 1996.

[7] O. M. Ramahi, "Complementary operators: A method to annihilate artificial reflections arising from the truncation of the computational domain in the solution of partial differential equations," IEEE Trans. Antennas Propagat., vol. 43, pp. 697-704, July 1995.

[8] - "The concurrent complementary operators method for FDTD mesh truncation," IEEE Trans. Antennas Propagat., vol. 46, pp. 1475-1481, Oct. 1998.

[9] O. M. Ramahi and J. Schneider, "Comparative study of the PML and C-COM mesh-truncation techniques," IEEE Microwave Guided Wave Lett., vol. 8, pp. 55-57, Feb. 1998.

[10] Y. P. Chiou and H. C. Chang, "Complementary operators method as the absorbing boundary condition for the beam propagation method," IEEE Photon. Technol. Lett., vol. 10, pp. 976-978, July 1998.

[11] D. Jiménez and F. Pérez-Murano, "Improved boundary conditions for the beam propagation method," IEEE Photon. Technol. Lett., vol. 11, pp. 1000-1002, Aug. 1999.

[12] C. T. Law and X. Zhang, "Concurrent complementary operator boundary conditions for optical beam propagation," IEEE Photon. Technol. Lett., vol. 12, pp. 56-58, Jan. 2000.

[13] P. L. Liu, Q. Zhao, and F. S. Choa, "Slow-wave finite-difference beam propagation method," IEEE Photon. Technol. Lett., vol. 7, pp. 890-892, Aug. 1995.

[14] F. Ma, "Slowly varying envelope simulation of optical waves in time domain with transparent and absorbing boundary conditions," J. Lightwave Technol., vol. 15, pp. 1974-1985, Oct. 1997.

[15] J. Shibayama et al., "Efficient time-domain finite-difference beam propagation methods for the analysis of slab and circularly symmetric waveguides," J. Lightwave Technol., vol. 18, pp. 437-442, Mar. 2000.

[16] M. Koshiba, Y. Tsuji, and M. Hikari, "Time-domain beam propagation method and its application to photonic crystal circuit components," $J$. Lightwave Technol., vol. 18, pp. 102-109, Jan. 2000. 